\title{
Le tournant instrumental des politiques culturelles
}

\author{
Emmanuel Négrier \& Philippe Teillet
}

\section{Résumé :}

Cet article, relatif aux politiques culturelles de l'État et des régions, examine l'hypothèse de changements résultant moins de transformations idéelles que de la mise en œuvre d'instruments nouveaux. Les discours sur ces politiques, les ambitions que leur donnent les dirigeants politiques sont en effet frappés d'une grande inertie. En revanche, les interventions culturelles publiques sont marquées depuis quelques années par l'introduction de normes de gestion ou de dispositifs d'intervention relativement inédits. Il s'agira donc de mesurer l'impact des changements qui peuvent en résulter. La première partie traite des instruments de la réforme de l'État et à leur impact sur ses services culturels déconcentrés au niveau régional. La seconde partie se concentre sur les instruments déployés par les régions dans le cadre de leurs propres politiques culturelles.

Mots clefs : Politiques culturelles, décentralisation culturelle, réforme de l’État, instrumentation, régionalisation

\section{Abstract}

This article, concerning State and Regions cultural policies, examines the hypothesis of changes resulting fewer ideals transformations than of new instruments implementation. The discourses on these policies, the ambitions which give them the political leaders are indeed struck by a big slowness. On the other hand, the public cultural interventions are marked since a few years by the introduction of standards of management or relatively unpublished plans of intervention. It will thus be a question of measuring the impact of the changes which can result from it. The first part handles instruments of the State reform and in their impact on its cultural departments decentralized at the regional level. The second part focuses on the instruments implemented by the Regional Councils in the context of their own cultural policies.

Keywords: cultural policies, cultural decentralization, State reform, policy tools, regionalization

Lors d'un exercice de prospective assez exceptionnel, le ministère français de la culture et de la communication s'est attaché il y a quelques années à construire plusieurs scénarios pour le devenir des politiques culturelles (DEPS 2011). Trois dynamiques y jouent un rôle fondamental : la globalisation, la mutation numérique, le rapport entre individualisme et société. Selon cette analyse, le rôle du ministère se réduira inéluctablement. Les acteurs du marché des produits culturels, les autorités locales, l'Union européenne et les grands opérateurs publics occuperont désormais une place telle que le ministère ne pourra plus prétendre revendiquer la conduite des affaires culturelles. Par ailleurs, en rappelant que s'il n'a jamais été nettement circonscrit, le territoire de ces politiques s'est largement étendu, cette prospective considère qu'il sera de plus en plus difficile de détacher les affaires culturelles de questions technologiques, diplomatiques, éducatives (entre autres) relevant d'autres administrations, voire d'autres entités politiques.

Ces analyses confirment et prolongent de multiples diagnostics pointant des changements en cours au sein des politiques culturelles publiques. Et pourtant, faute de pouvoir proposer un nouveau récit capable d'en synthétiser le sens et d'en légitimer efficacement l'existence, la perspective d'une démocratisation de la culture paraît en constituer l'inusable référentiel. Certes, le paradigme de la diversité culturelle, challenger récent, lui conteste sa domination 
(Bonet et Négrier 2008). Cette philosophie d'action alternative trouve sa force principale dans le soutien d'organisations internationales (Unesco, Union européenne) et d'Etats qui entendrent garantir à l'échelle mondiale un pluralisme que menacent sans cesse de forts déséquilibres économiques. La question de la diversité culturelle s'appuie également sur la situation d'Etats plurinationaux ou fortement régionalisés qui trouvent en elle de quoi fonder ou régénérer leur ordre social et politique. Mais, en France, elle est loin de pouvoir bénéficier du soutien d'un groupe social potentiellement dominant dans ce champ d'intervention, de médiateurs (Jobert \& Muller 1987) capables de porter un changement de référentiel. A l'inverse, la démocratisation culturelle est forte de son articulation avec des valeurs et principes politiquement puissants dans ce pays (Ritaine 1983) : l'unité culturelle de la nation et le rôle de l'action publique pour l'égalisation des conditions sociales. Elle offre ainsi à ses promoteurs et soutiens (professionnels de la culture, élus, services de l'Etat et des collectivités territoriales) des profits symboliques et politiques que la diversité culturelle ne peut promettre. En revanche, cette dernière impose de rompre avec des conceptions routinisées des politiques culturelles, d'assumer les coûts matériels et symboliques de la sortie du «chemin de dépendance » de la démocratisation d'autant plus contraignant que cette dernière a bénéficié depuis au moins 50 ans de larges soutiens politiques et professionnels. Or, le contexte économique et budgétaire menaçant dans lequel se déploient aujourd'hui ces politiques invite à la prudence ceux qui prétendent en prendre la responsabilité. Plutôt que de devoir faire face aux aléas de ruptures politiques et symboliques, ils préfèrent la préservation de cet «étendard» (Dubois, 1999) qu'est devenue la "démocratisation culturelle » et se garantir ainsi le soutien des groupes sociaux et organisations professionnelles qui y sont le plus attachés.

Il est donc peu contestable que les politiques culturelles connaissent des évolutions. Mais ces changements ne peuvent être interprétés comme le passage d'un référentiel à un autre. Une forme d'inertie discursive et idéologique les marque plutôt. Des rapports de forces relativement stabilisés assurent aux représentations que les groupes dominants ont de l'action culturelle publique une position encore très solide. Dans ces conditions, les mutations observables relèvent plus de l'hybridation tendancielle ou du non-dit quand des valeurs ou priorités sont politiquement (ré)affirmées, alors qu'elles sont plus ou moins profondément affectées par des décisions de moyenne portée, notamment budgétaires, passablement contradictoires et non nécessairement cohérentes.

C'est pourquoi, l'analyse de changements au sein des politiques culturelles françaises doit se détourner du terrain (sur)symbolique où une tradition de grandiloquence en la matière les a installées de longue date. Elle invite au contraire à porter une attention plus fine à la façon dont elles sont concrètement conduites et mises en œuvre. C'est ce que permet notamment l'examen des instruments de politiques publiques. Il propose en effet de privilégier les dispositifs « à la fois technique(s) et (sociaux) qui organise(nt) des rapports sociaux entre la puissance publique et ses destinataires en fonction des représentations et des significations dont il(s) (sont) porteur(s)» (Lascoumes, Le Galès, 2004a : 267). L'intérêt d'une telle approche est triple : ces instruments peuvent produire des effets indépendants des objectifs affichés ; ils ont, par delà leur finalité fonctionnelle, des dimensions politiques que l'analyse révèle ; enfin, on y repère des changements de politiques publiques passant moins par les effets attendus que par les « recettes » employées.

C'est au regard de ces perspectives analytiques que deux séries d'instruments de politiques culturelles seront étudiées dans ce travail. La première s'inscrit dans le cadre d'une réforme de l'Etat réactivée au début des années 2000 et qui va s'impacter sur son administration 
culturelle. Les instruments en cause ici relèvent de la nouvelle gestion publique et d'une conception de l'administration fortement orientée par le modèle de référence que constitue le management des entreprises privées (Bezes 2009). A la différence des instruments habituellement observés dans l'analyse des politiques publiques - qui interviennent dans « les relations entre la société politique (via l'exécutif administratif) et la société civile (via ses sujets administrés) »(Lascoumes, Le Galès, 2004b, 21) -, les relations retenues dans cette étude se déploient à l'intérieur de l'exécutif. Il s'agit principalement de celles qui s'établissent entre services centraux du ministère de la culture et services déconcentrés, les Directions Régionales des Affaires Culturelles (DRAC). Cette réforme de l'État repose principalement sur une nouvelle organisation des lois de finances, la LOLF 1 , ainsi que sur une politique dite de «modernisation» de l'administration d'Etat, la RGPP2 ${ }^{2}$. A ces deux instruments phares s'ajoute de façon plus discrète mais particulièrement sensible au sein des services de l'État, un logiciel, Chorus, permettant de gérer l'ensemble des processus financiers et comptables. La mise en œuvre de ces dispositifs et outils se traduit par une diffusion à partir du pouvoir central de règles pratiques redéfinissant les activités et parfois même le métier - comme ensemble de pratiques professionnelles - des agents des services déconcentrés. C'est pourquoi, l'objet de cette contribution sera pour partie d'étudier les effets des instruments de la réforme de l'État dans le champ des politiques culturelles.

Nous nous intéresserons également aux instruments mobilisés par les autorités locales. Notre regard concernera alors les régions. Il ne s'agit pas des acteurs publics les plus influents en matière culturelle. En 2006 leurs dépenses culturelles représentaient 168 millions d'euros pour 407 millions s'agissant des départements et 968 millions pour les communes de plus de 10000 habitants. Toutefois, on peut faire l'hypothèse qu'au regard de cette faiblesse, les régions ont développé des instruments de politiques culturelles singuliers distinguant de plus en plus leur action en ce domaine de celle des autres collectivités publiques. Caractérisée par une forte propension à croiser leurs dépenses avec celles d'autres collectivités ou de l'Etat, les régions souffrent en effet d'un défaut de lisibilité alors que le territoire régional est de plus en plus souvent présenté comme un cadre pertinent pour envisager une recomposition des politiques publiques de la culture. La prospective ministérielle précédemment cité a d'ailleurs envisagé dans tous ses scénarios la montée en puissance culturelle des régions. Il semblait donc important de s'intéresser aux dispositifs qu'elles tendent à mobiliser en ce domaine afin d'y repérer (ou non) les changements annoncés.

Tous ces instruments partagent un air de famille, à laquelle on peut d'ailleurs rattacher les réglementations européennes sur les aides publiques (Paquet Monti-Kroes, puis Almunia de 2011) dont l'impact restrictif et re-centralisateur des politiques culturelles est posé (Lucas 2012). Malgré leurs différences (du côté de l'Etat, ils sont objectivés dans des textes - Loi organique, programme gouvernemental de réforme administrative - et des organisations administratives; du côté des régions, ils apparaissent plutôt au travers de pratiques convergentes sans mise en forme globale), ces instruments relèvent de nouvelles modalités d'administration des affaires publiques où se mêlent de façon particulièrement complexe et parfois contradictoire, des objectifs de rationalisation et de démocratisation. Rigueur et

\footnotetext{
${ }^{1}$ Loi organique $\mathrm{n}^{\circ} 2001-692$ du $1^{\text {er }}$ août 2001 relative aux lois de finances.

2 Révision Générale des Politiques Publiques : programme de «modernisation » de l'action de l'État touchant l'ensemble des politiques publiques et l'ensemble des ministères lancé en juin 2007. Son ambition est de réduire les charges de l'État, en particulier par une forte réduction du nombre de ses agents, tout en instaurant de nouvelles méthodes de gestion visant à plus d'efficacité. Depuis 2012, la Modernisation de l'Action Publique (MAP) a pris la suite de la RGPP avec l'ambition de réorienter la réforme de l'Etat. Son implémentation, ultérieure à nos enquêtes, a néanmoins fait l'objet d'investigations complémentaires.
} 
performance, d'un côté, participation et concertation de l'autre, sont des impératifs auxquels tous les instruments étudiés ici doivent permettre de mieux répondre. Mais la situation des régions est nettement moins problématisée et politisée que l'introduction de la nouvelle gestion publique au sein des services de l'Etat. Cette dernière a en effet été perçue comme capable d'affecter le bilan de plus de 50 ans d'action du ministère de la culture ${ }^{3}$. Dans un contexte de restrictions budgétaires récurrentes, la référence à la gestion privée tend à être entendue comme l'annonce d'une réduction du périmètre d'intervention de l'Etat et, plus largement, de son influence en matière culturelle. Le devenir de ses services déconcentrés et la recentralisation possible du ministère apparaissent alors comme le signe de son désengagement non seulement territorial mais aussi potentiellement sectoriel. Le dévoilement d'intentions réelles ou supposées des autorités nationales, en contradiction avec la célébration rituelle de l'importance des enjeux culturels, devient un thème récurrent des mobilisations syndicales et professionnelles du secteur. On mesure alors l'impact des transformations du rapport entre administrations centrales et déconcentrées qui excède très largement les frontières des services de l'Etat. Le jeu des régions est en revanche une question beaucoup moins chaude politiquement. Elle s'inscrit néanmoins parmi ces luttes plus ou moins sourdes qui opposent les catégories de collectivités territoriales alors que leur réforme et la possible disparition (ou effacement progressif) d'un ou de plusieurs échelons d'intervention se maintient à l'agenda des gouvernements depuis plusieurs décennies.

Le tournant instrumental des politiques culturelles consiste donc à examiner l'hypothèse de changements importants concernant les politiques de l'État et des régions qui doivent moins à des évolutions de leurs référentiels qu'à la mise en œuvre en ce domaine d'instruments nouveaux dans la gestion des services et la relation des administrations à leurs ressortissants. Dans une première partie, nous nous intéresserons aux instruments de la réforme de l'État et à leur impact sur ses services culturels déconcentrés au niveau régional. Dans une seconde partie, nous observerons les instruments déployés par les régions dans le cadre de leurs propres politiques culturelles 4 .

\section{L'Etat territorial face aux outils de la nouvelle gestion publique}

La déconcentration du ministère de la culture a été difficilement acquise en raison des oppositions qu'elle a dû surmonter à la fois à l'intérieur du ministère et à l'extérieur (les professionnels de la culture ayant longtemps craint tant la perte d'influence en ce domaine de l'État central, que celle corrélative des profits symboliques que ce dernier pouvait leur accorder) (Négrier, Teillet, 2014). Difficilement acquise, la reconnaissance de l'État culturel territorial paraissait fragile et mériter un militantisme de tous les instants. C'est au regard d'un tel contexte qu'il faut apprécier l'impact de la réforme de l'État, à l'œuvre depuis plus d'une décennie et inspirée du Nouveau Management Public - NMP - (Bezes 2009). En s'appuyant sur des forces centralisatrices toujours actives, mettra-t-elle fin à cette évolution ?

\footnotetext{
3 Voir par exemple les manifestes des syndicats et organisations professionnelles du spectacle vivant ciblant prioritairement la RGPP : http://www.syndeac.org/index.php/communiques-publics/632-communique-sur-lesmultiples-tentatives-de-destabilisation-qui-menacent-le-secteur-du-spectacle-vivant-et-les-artistes-28-octobre2010 et http://www.syndeac.org/assets/POLITIQUES_PUBLIQUES/encart_liberation_avril2010.pdf

4 Ce travail résulte de deux enquêtes. La première est une comparaison de 4 politiques culturelles régionales (Négrier \& Teillet 2011). La seconde s'est appuyée sur deux Directions Régionales des Affaires Culturelles (DRAC).
} 


\subsection{Usual suspects ou les instruments potentiels de reconcentration : LOLF, RGPP et Chorus}

La LOLF réforme en profondeur la gestion de l'État, découpée en 34 missions, 133 programmes et près de 580 actions. Il est attendu d'elle un meilleur contrôle parlementaire de l'efficacité de la dépense publique. Chaque année, l'administration doit expliquer ses objectifs et sa stratégie et rendre compte de son action. A la tête de chaque programme, un responsable est doté d'une plus grande liberté dans la gestion de ses moyens pour atteindre les objectifs votés par le Parlement. Il s'appuie sur des relais opérationnels qui disposent des mêmes souplesses de gestion pour que chaque euro dépensé soit plus utile et plus efficace. Cette liberté de gestion est la contrepartie d'un engagement sur des objectifs de performance : pour chaque objectif, des indicateurs concrets doivent permettre de mesurer les résultats des actions menées. La LOLF va donc se traduire par la production d'un certain nombre d'indicateurs (Programmes Annuels de Performance) et de rapports d'activités (Rapports Annuels de Performances) qui privilégient le plus souvent des dimensions quantitatives et donc chiffrées. C'est pourquoi, au sein du ministère de la Culture, la LOLF vient en opposition frontale avec une culture administrative fondée sur la qualité (plus que les quantités) et sur la revendication d'une exception pour des catégories d'interventions particulièrement rétives à leur mise en chiffre.

De son côté, la Révision Générale des Politiques Publiques (RGPP) se proposait de mettre à plat les politiques de l'Etat de façon à les rendre à la fois plus efficaces et moins coûteuses (notamment par le non remplacement d'un fonctionnaire sur deux partant à la retraite). Les personnels des DRAC ont pu se sentir relativement épargnés par la RGPP dans la mesure où, se traduisant par la refonte des services de l'État en région en ne laissant subsister de la trentaine de structures étatiques préexistantes que 7 directions régionales (en plus des rectorats), les DRAC ont été relativement épargnées. Elles n'ont en effet pas eu à intégrer des directions régionales nouvelles, à régler toutes sortes de difficultés organisationnelles, voire identitaires. Plus encore, elles se sont étoffées des services départementaux de l'architecture et du patrimoine (SDAP, devenus Services Territoriaux, STAP). Mais la RGPP se traduisait aussi par des réorganisations et mutualisations de services, ainsi que par des tensions sur les emplois disponibles qui affectent très sensiblement et souvent négativement le travail des agents. Le changement principal se situant au sein des services financiers dans le traitement des opérations de paiement et, en particulier, dans la gestion des subventions que les DRAC attribuent. La nouvelle organisation limite en effet leur action à l'instruction des demandes et décisions de subvention. Les opérations suivantes, en particulier le versement des sommes prévues (activité d'ordonnateur secondaire), sont désormais mutualisées dans le cadre de plateformes régionales.

Pour les responsables des DRAC, cette situation paraît moins résoudre des problèmes qu'en poser de nouveaux. Les personnels précisent d'ailleurs que les difficultés sont liées à la coïncidence de la mise en œuvre de la RGPP avec celle du logiciel Chorus, l'outil intégré de gestion financière, budgétaire et comptable destiné à l'ensemble des administrations de l'État, aux plans central et déconcentré. Il a été déployé par vagues d'administrations d'Etat entre 2010 et 2012. Les défauts et difficultés d'application de Chorus ont été officiellement et publiquement dénoncés 5 . Loin de gagner en efficacité, des retards de paiement placent les services des DRAC dans une grande difficulté vis-à-vis des structures qu'elles soutiennent et qui sont fragilisées par des délais de paiement sensiblement allongés, à telle enseigne que

\footnotetext{
5 Cf. le rapport annuel 2011 de la Cour des Comptes, p. 267-294. http://www.ccomptes.fr/fr/CC/documents/RPA/P_chorus.pdf
} 
certains évoquent même l'hypothèse que la complexification des procédures pourrait correspondre à un projet politique : celui d'endiguer l'accroissement du nombres d'acteurs légitimes à un soutien public. Les défauts de Chorus donnent lieu à de multiples arbitrages, corrections et rectificatifs (Négrier \& Teillet 2014).

\subsection{La déconcentration culturelle, fin ou suite?}

Ce qui se passe dans le cadre du ministère de la culture est une illustration exemplaire de la distance qui sépare l'esprit des réformes et leur traduction concrète. Loin d'accroître la responsabilité des agents sur les budgets qui leur sont confiés, la LOLF est perçue par ces derniers comme s'étant plutôt traduite par la réduction de leur autonomie. Les promesses de cette nouvelle organisation budgétaire ne seraient pas tenues, tant au regard des objectifs affichés par ses promoteurs que des expérimentations qui avaient précédé sa généralisation. Est-ce alors la fin du mouvement de déconcentration, voire le retour à une certaine centralisation?

\section{La déconcentration culturelle : le scénario de la fin}

C'est ce que semble illustrer le programme Création où des mécanismes essentiellement politiques soutiennent des pratiques à rebours des perspectives de la LOLF. De façon générale, les capacités de mobilisation et de médiatisation du spectacle vivant, au sein de ses institutions ou événements les plus emblématiques, justifient une attention particulière des pôles politiques de la Centrale (Directeurs généraux, secrétariat général, cabinet) qui souhaitent avoir la main sur la gestion des dossiers sensibles du secteur, tout particulièrement sur les questions financières. Formellement, la déconcentration reste donc acquise. Substantiellement, elle est écornée, puisque la part variable, qui mobilise les efforts des acteurs, échappe aux DRAC. Ainsi, la Centrale a relancé un mécanisme d'aide exceptionnelle, ciblée, déclenchée directement depuis la Direction vers l'acteur individuel, sans passer par le «droit commun » de la feuille de route donnée aux DRAC. De la sorte, celles-ci deviennent, pour le bénéficiaire (une Scène nationale, une compagnie conventionnée, un Centre chorégraphique, etc.) une sorte d' "ordinaire » qu'il convient d'enrichir par une stratégie spécifique, tournée vers l'administration centrale.

De son côté, le programme «Transmission » témoigne d'un processus spécifique, celui de l'appel à projet, se traduisant également par une perte de responsabilité des DRAC. Ce programme correspond à des domaines d'action très divers. On y trouve d'abord ce que l'on nomme aussi l'action culturelle, qu'il s'agisse des relations avec les acteurs de l'Éducation populaire, de l'intervention culturelle en milieux sociaux, zones sensibles, hôpitaux, prisons. On y trouve aussi tout ce qui concerne l'éducation artistique, soit les interventions en milieu scolaire. Parallèlement, figurent le financement des pratiques amateurs, des conservatoires, des arts de la rue, des résidences artistiques, le fonctionnement général de la direction régionale. La procédure comprend le lancement de l'appel à projet régional, la sélection des réponses en région, l'instruction des réponses en transversalité avec les autres services en région, la remontée puis le traitement en Centrale des dossiers, enfin, la redescente des crédits sur dossiers acceptés. Tout ce processus aboutirait à peu près aux mêmes résultats si le conseiller à l'action culturelle était chargé de répartir directement l'enveloppe. Le coût de traitement du projet serait ainsi fortement diminué. La question de l'efficience n'est donc pas prioritaire. Bien au contraire, cette centralisation des dossiers induit des modes de régulation proprement nationaux, qui se détachent nécessairement des terrains. La reconcentration (axe vertical) a pour conséquence d'induire un resserrement (axe horizontal) du ministère sur luimême, y compris dans les domaines où il s'appuie pour exister sur une interdépendance avec 
d'autres administrations ou les collectivités territoriales. On ne peut plus claire contradiction entre objectifs, modalités de mise en œuvre et principe d'efficience.

\section{La déconcentration culturelle : épisode « $2 » ?$}

Le diagnostic fortement répandu dans les DRAC, d'une reconcentration des pouvoirs au sein du ministère de la culture, repose largement sur une transformation à marche forcée de leurs pratiques. Après avoir pu bénéficier d'une certaine reconnaissance de leur légitimité, la suite de réformes ici étudiée (LOLF, RGPP, Chorus) est perçue comme la mise en cause de ces fragiles et récents acquis. Toutefois, ce gouvernement des conduites ne parvient pas à écarter tout jeu, voire toute réappropriation de ces instruments, une forme de latitude permettant à certains agents de retrouver un espace d'autonomie s'appuyant sur leur capacité à être moins les jouets des instruments précédemment évoqués qu'à parvenir à en jouer. Ce n'est en effet pas tant le cadre normatif et financier de la déconcentration qui est remis en cause, que la manière dont sont encadrés les moyens, les procédures et les agents. Nos observations permettent de souligner des différenciations fines dans la mise en œuvre des instruments de rationalisation de l'action publique au sein d'un même département ministériel. Cette situation repose à la fois sur la politisation et la dépolitisation de certains enjeux. Les secteurs très professionnalisés (et ainsi relativement dépolitisés, comme le patrimoine) peuvent être épargnés par cette rationalisation. Point n'est besoin ici de piloter à distance un secteur où la reconcentration est dans les mentalités (mécanisme de gouvernementalité), dans le réseau diversifié des procédures et valeurs communes. A l'inverse, la médiatisation des grandes crises marquant le spectacle vivant lui garantit un interventionnisme beaucoup plus clair du pôle politique de la Centrale. Une différenciation s'institue alors entre une gestion déconcentrée des ressources ordinaires et l'activation centrale de ressources sélectives, de primes, de fonds ciblés, etc. (mécanisme de bipolarisation). Enfin, dans certains domaines, la décision est déportée d'un lieu à l'autre du spectre d'action publique, aux fins de contrôle de la substance en question (mécanisme de gouvernement à distance).Il n'est pas interdit de penser que, au sein d'un même ministère, la coexistence de plusieurs orientations du contrôle soit applicable à d'autres administrations que celle de la Culture.

On observe également que chez certains agents des DRAC, la nécessité de rendre des comptes va de pair avec l'acquisition de nouvelles compétences telles que savoir jouer stratégiquement avec ces outils (au mieux de leurs intérêts ou convictions), et ce, tant vis-à-vis de la Centrale que de leurs partenaires territoriaux. Une certaine rationalisation de leurs actions est en outre souvent présentée comme un élément positif dont, sur la durée, les effets s'inscrivent dans les perspectives de missions de contrôle et d'expertise qui constituent leur cœur de métier. Ceci contrastant avec les effets bien plus contraignants et jugés plus souvent négatifs de la RGPP et de Chorus. En outre, les effets de contrainte des indicateurs promus en Centrale doivent être précisés et nuancés. Censés permettre de porter un jugement éclairé sur l'action des services, ils sont en réalité moins contestés dans leur principe qu'à travers leur faible pertinence (voire leurs contradictions). Il convient cependant de distinguer les indicateurs entre eux, selon deux catégories. La première concentre les indicateurs concernant l'ensemble des DRAC. De l'aveu général, leur intérêt est faible, dans la mesure où ils ont échappé (dans leur construction) à toute discussion réelle. Ces indicateurs servent au contrôle vertical que les DRAC subissent de la part de la Centrale (elle-même sous contrôle du ministère du Budget), ainsi qu'à la présentation des bilans annuels devant le Parlement. Vus par les agents des DRAC, ils constituent un pur exercice de style. La seconde catégorie est celle que les services élaborent eux-mêmes à l'occasion de la définition de leurs objectifs. Ils sont présentés et discutés au sein des réunions bilatérales avec la Centrale et sont ensuite intégrés dans la «feuille de route », soit le «Mandat relatif à la révision des critères d'intervention de l'État 
dans le domaine de la création, période 2011-2013 ». Si le développement des indicateurs dans l'activité des personnels des DRAC est sensible, ceux qui à leurs yeux ont une utilité supérieure sont justement ceux à la production desquelles ils ont pu contribuer.

Enfin, par delà leur impact en termes de productivité financière, l'application de ces réformes se traduit par l'influence renforcée des instances centrales du Ministère, mais aussi par celle d'acteurs jusque là assez marginaux que sont les préfectures de région. Les problèmes posés par la mise en œuvre simultanée de ces réformes favorisent une intégration verticale des services de l'État (échanges avec la Centrale sur la mise en œuvre de tous ces changements), mais aussi horizontale (entre services déconcentrés d'une même région) par une forme de connaissance et de reconnaissance de la situation des uns par les autres, voire une certaine solidarité dans l'épreuve 6 . Dès lors, on observe, en plus des mécanismes précédemment décrits, la structuration régionale de l'État territorial. En ce sens, il y aurait une reconcentration régionale du pouvoir d'État marquée par une intensification des relations préfectures de région - DRAC. Cette intensification fait réellement entrer l'administration préfectorale dans un jeu à la fois horizontal (avec la DRAC) et vertical (avec les instances centrales de ministères ou les préfectures de département) qui n'est pas exempt de rapports de force. Complétée par les mobilités accrues des personnels vers d'autres services territoriaux de l'État, émerge en matière culturelle une forme, régionale de reconcentration ou, pour utiliser un terme encore plus barbare, de «re-déconcentration», marquée par un redéploiement de ressources entre acteurs déconcentrés, qui peut donner aux préfets de région une capacité d'influence plus nette sur les politiques du ministère lui-même.

C'est pourquoi, au regard de l'hypothèse d'une fin de la déconcentration, la situation oscille entre un regain de puissance des échelons centraux - qui paraît signaler l'achèvement d'une structuration originale des politiques culturelles - et une redistribution des cartes, impliquant autrement les cadres territoriaux et semblant plutôt plaider pour un changement de vocation du modèle déconcentré, une autre «fin », donc. Dans cette perspective, le programme MAP (Modernisation de l'Action Publique) qui a pris la suite de la RGPP après l'alternance de 2012, ne constitue qu'un changement épiphénoménal. Son originalité initiale reposait sur une méthode plus concertée, moins abrupte, plus sensible à l'évaluation initiale et moins exclusivement centrée sur les réductions d'effectifs que la réforme précédente. Nos premières observations relativisent toutefois la portée d'un tel changement, qui s'avère plus discursif et artificiel que substantiel. Cette analyse reste cependant à faire de façon systématique.

\section{L'instrumentation des politiques culturelles régionales}

Les interventions des régions en matière culturelle tendent à adopter un style particulier qui vient précisément des instruments spécifiques qu'elles mobilisent. Deux catégories d'instruments seront ici prises en compte. Les premières sont leurs ressources d'expertise en matière culturelle et les agences qu'elles ont créées. Les seconds concernent la structuration d'un dialogue régional avec les organisations du secteur.

L'observation du développement de ces politiques régionales montre en effet qu'il s'inscrit dans une organisation polycentrique de l'intervention des pouvoirs publics. Acteur le plus récent et financièrement le moins doté, les régions ont très tôt cherché à marquer leur territoire culturel. Mais en croisant leurs financements avec les autres collectivités publiques et sur les mêmes objets d'intervention, elles ont longtemps donné une faible visibilité à leurs

\footnotetext{
6 L'Opérateur National de Paye (ONP) constitue un 4ème instrument de reconcentration dans la mesure où il traite la paye de tous les agents à partir des données de gestion transmises par les ministères. Cf. http://www.fonctionpublique.gouv.fr/article961.html
} 
actes. En revanche, commence à poindre un choix d'instruments d'intervention particulier qui tend à doter d'une morphologie particulière leurs politiques en matière culturelle.

La domination de l'État sur les questions culturelles, qui a marqué la France depuis l'orée des années soixante jusqu'à la fin des années quatre-vingts environ, a en effet laissé place à une situation concurrentielle, impliquant les différents niveaux d'action publique. Les grandes villes présentent des ressources suffisantes pour peser considérablement sur la définition des politiques culturelles. Mais ces dernières sont aussi le produit de leurs interactions avec les autres collectivités publiques, ainsi qu'avec les professionnels de la culture, leurs organisations et la société civile mobilisée en ce domaine. Ce jeu d'alliances et de coopération est aussi un espace de confrontations et de différenciation. Il s'agit alors de cofinancer tout en se distinguant, d'imprimer sa marque tout en coopérant. Cet enjeu a été particulièrement sensible pour les régions, dernières venues (avant le récent développement des intercommunalités) dans le paysage institutionnel français.

Existe-t-il, alors, un style proprement régional de politique culturelle, et si oui, selon quel critère l'appréhender ? La tentation substantielle qui voudrait que le style dépende au fond d'une culture préalable, d'une identité régionale spécifique, est sans doute une fausse piste (Négrier, Teillet, 2011). Les régions ont longtemps plutôt fondé leurs interventions culturelles sur leur différence mais aussi leur complémentarité vis-à-vis des villes et surtout des départements. Si ces derniers concentraient une part importante de leurs budgets culturels sur le patrimoine (dans une conception large), les régions optaient stratégiquement pour des actions où elles étaient susceptibles de marquer leur différence : le spectacle vivant, en particulier la musique et la danse, ainsi que, mais dans une moindre mesure, le cinéma (production et diffusion). Mais en croisant leurs financements avec ceux d'autres collectivités publiques et parce qu'une action culturelle est toujours localisée dans une ou plusieurs villes captant à leur profit les bénéfices symboliques susceptibles d'en être tirés, les régions ont progressivement adopté de nouvelles façons de légitimer leurs politiques culturelles.

Une première a consisté à encastrer leurs politiques dans leurs grands domaines d'attributions. Cette orientation avait été bien repérée par Mireille Pongy (2004 : 52) qui observait, par exemple, que les dispositifs de ce type, visant à soutenir financièrement la «demande culturelle » des lycéens, prolongeaient la responsabilité des régions à l'égard des lycées. Il en va de même de leurs interventions en faveur de l'économie régionale du livre ou de la formation dans le champ du spectacle vivant qui participent de leurs principaux domaines de compétences (l'économie et la formation professionnelle). Mais, par delà cette articulation intersectorielle, les régions tendent aujourd'hui à mobiliser deux instruments particuliers susceptibles de plus nettement différencier leurs interventions en ce domaine.

\subsection{La construction d'une expertise au sein des agences culturelles régionales}

Les DRAC ont longtemps eu le monopole de l'expertise culturelle régionale fondée sur leurs personnels scientifiques (dans le champ patrimonial) et leurs conseillers sectoriels traduisant en région les orientations de leurs directions ministérielles. Leurs personnels ont été impliqués dans les coopérations institutionnalisées entre l'État et les régions au sein des FRAC, FRAM et FRAB ${ }^{7}$, mais aussi des Centres Régionaux du Livre (CRL) et des Associations Musique et Danse (sur le modèle de structures identiques créées à la suite du Plan Landowski -1970dans le cadre des départements). L'impulsion de l'État a permis de créer des structures de ce type dans la plupart des régions afin de les associer aux politiques ministérielles dans les domaines musicaux et chorégraphiques. Elles sont progressivement devenues des outils des

7 Fonds Régionaux d'Art Contemporain, Fonds Régionaux d'Acquisition des Musées, Fonds Régionaux d'Acquisition des Bibliothèques. 
régions qui assurent la majorité de leurs financements même si l'État est généralement toujours représenté dans leurs conseils d'administration. Ces agences régionales, le plus souvent à statut associatif, complètent ainsi, comme au niveau des départements, les services des régions et prennent en charge une fonction d'interface avec les milieux et professionnels de la culture intervenant dans leurs champs d'attribution en visant tout particulièrement des missions d'information, de formation, de structuration et de coordination. Certaines (environ 5 sur 20) ont connu ces dernières années un mouvement de recomposition lié à des fusions et/ou des élargissements de leurs domaines. L'existence de structures spécialisées dans le secteur des musiques actuelles (pôles régionaux) ou des musiques traditionnelles, celle d'agences de conseil et de formation pour la gestion d'entreprises culturelles puis d'observatoires régionaux, a suscité, en particulier lors de crises structurelles ou politiques, une redéfinition de ces agences selon des formules variables laissant parfois séparé ce qui ailleurs était réuni.

Ce processus d'agencification au sein des politiques culturelles régionales a donné naissance à un regroupement des structures concernées au sein d'une association professionnelle: la Plate-forme interrégionale d'échange et de coopération pour le développement culturel. Une étude menée au près de ses membres 8 indiquait, en 2008 , que leurs salariés représentaient un effectif total de 259 contrats à durée indéterminée (CDI), pour 235 équivalents en temps plein (ETP). Les agences culturelles régionales revendiquent ainsi une fonction d'expertise sur leurs champs d'intervention et en particulier sur le spectacle vivant là où les DRAC ne peuvent aligner le plus souvent que leurs conseillers «musique et danse » et «théâtre ». Cette expertise se traduit par des activités de conseil, d'accompagnement à la structuration des milieux professionnels, d'information, d'observation et de ressources, de concertation et de coordination et de formation professionnelle. Si elles se heurtent parfois à une mise en cause de la part de secteurs professionnels contestant leurs méthodes et compétences et revendiquant à la fois plus d'autonomie dans certaines activités d'observation et un rapport direct aux autorités régionales (sans passer par le filtre de ces agences), ces structures ont vocation à représenter une dimension spécifique des interventions culturelles des régions mettant sur pied ces agences et captant à travers elle une part de l'expertise que les services de l'État ne peuvent plus assumer ou plus assumer seuls dans certains domaines.

\footnotetext{
8 Nous remercions Stéphane Grosclaude, son directeur, de nous l'avoir communiquée.
} 
La portée légitimante de cette expertise est toutefois doublement limitée. D'abord par le degré d'écoute des responsables politiques régionaux qui peuvent préférer répondre à des logiques politiques plutôt que de mobiliser les outils de rationalisation que leur proposent ces agences. Ensuite, par les milieux culturels professionnels qui peuvent aussi se placer sur un registre politique ou imposer leur propre expertise tirée de capacités d'auto-observation. Par ailleurs, ces agences souffrent souvent de ne pas trouver la bonne distance à l'égard des exécutifs régionaux. Trop éloignées et extérieures, elles peinent à se faire entendre ; trop intégrées, elles risquent de se transformer en (ou d'être considérées comme) outils de valorisation de l'action régionale. Deux perspectives se dessinent alors pour les agences culturelles régionales. Selon la première, leur morcellement et hétérogénéité, les luttes qui les opposent parfois aux services des régions, aux DRAC et aux organisations professionnelles, auraient bientôt raison d'elles, laissant au mieux subsister des structures moribondes. La seconde, plus optimiste, parie sur les besoins d'expertise dans la définition et la conduite des politiques publiques et sur la nécessaire institutionnalisation d'expertises territorialisées. Les instruments dont les régions se sont ainsi dotées constitueraient dès lors un incontestable coup d'avance.

\subsection{La structuration de dialogues avec la société civile en matière culturelle}

Face à un modèle français de politiques culturelles marqué par un fort volontarisme politique et la certitude que le débat démocratique doit s'arrêter aux limites de ce domaine, les politiques régionales opposent un penchant de plus en plus sensible à la concertation. Ce sont d'ailleurs fréquemment les agences précédemment évoquées qui ont pour mission d'organiser et d'animer, tout au long des années, les multiples assises, rencontres et autres journées d'études sur les champs plus ou moins vastes des politiques culturelles.

Espace intermédiaire entre le local et le national, mais qui a l'avantage par rapport aux départements d'être également celui des services déconcentrés du ministère, les régions sont assez rapidement apparues comme des échelons adaptés à la structuration des milieux culturels, à l'organisation de moments d'échanges entre acteurs et, surtout, de concertation avec les responsables politiques en charge de ces questions sur le territoire régional. Au début des années 2000 les interrogations sur les conditions de la gouvernance culturelle avaient conduit aux expériences des protocoles de décentralisation (visant à fixer, territoire par territoire, une règle du jeu entre les collectivités publiques impliquées), puis à partir de 2002, aux expériences régionales menées en Midi-Pyrénées et Lorraine qui devaient, comme pour les protocoles, déboucher sur des schémas de développement culturel proposant une meilleure répartition des rôles, une nouvelle définition d'objectifs communs et de propositions d'action. Si ces expériences trouvaient leur origine dans l'idée qu'elles pourraient contribuer à éclairer le législateur au moment de l'adoption de l'acte II de la décentralisation, leur temporalité et celle du travail législatif ont été nettement désajustées et la loi du 13 août 2004 adoptée avant que des enseignements aient pu être tirées de ces deux terrains. Il n'en reste pas moins que, dans ces deux régions, l'expérience s'est traduite par la constitution de « forums» ${ }^{9}$ culturels régionaux dont les participants ont apprécié l'existence et souhaité la pérennisation ${ }^{10}$.

Mais la généralisation de formes variées de concertations culturelles régionales doit moins à ces expérimentations qu'à la promotion de la participation au sein de la nouvelle grammaire d'action publique qui favorise l'ouverture des processus décisionnels tant pour renforcer la qualité technique des décisions que pour mieux en assurer l'acceptabilité. C'est ainsi que la

\footnotetext{
9 Au sens générique donné par B. Jobert (1992) de ce terme, soit de «lieux » ou de «scènes », plus ou moins institutionnalisés (structurés, durables) au sein desquels des acteurs ont des débats concernant une politique publique.

10 Nous remercions Mireille Pongy, chargée par l'Observatoire des Politiques Culturelles de Grenoble de suivre ces expérimentations, de nous avoir communiqué ses notes à ce sujet.
} 
formule des «Grenelle » s'est diffusée dans différents champs d'action et traduite en matière culturelle, plus particulièrement s'agissant du spectacle vivant, par les Entretiens de Valois ${ }^{11}$. Ceux-ci se sont conclus en janvier 2009 en préconisant notamment l'organisation de conférences régionales qui ont débuté en septembre de la même année en Rhône-Alpes. Parallèlement, le secteur des musiques actuelles avait suscité une concertation nationale en 2003 et 2004 qui avait aussi débouché sur un plan pour des politiques en ce domaine passant par l'organisation de concertations territoriales. Sans viser un échelon particulier les invitations faites aux DRAC et préfectures de région de se mobiliser sur ce thème ont fait de l'espace régional le cadre privilégié de ces concertations.

Toutefois, sur ce point également, il est nécessaire de souligner combien le changement peut parfois s'avérer modeste. Le puissant sentier de dépendance que le volontarisme politique constitue en matière culturelle n'épargne guère les régions et les autres types de collectivités territoriales. Le partage du pouvoir de décision, même sous les formes modestes d'une concertation, est un apprentissage difficile pour les élus comme pour les milieux culturels. Ces derniers sont en outre souvent marqués par de fortes concurrences internes et des déséquilibres tels entre acteurs et structures (du point de vue des moyens nécessaires pour participer à ces concertations, de leur dépendances à l'égard de leurs résultats, de leur capacité à s'y faire entendre et à y être pris en considération), qu'il leur est difficile de présenter un front uni et puissant capable de peser sur les orientations des politiques régionales.

\section{Conclusion}

Les instruments étudiés ici empruntent les uns et les autres au répertoire de pratiques caractéristiques de la nouvelle gestion publique, de la bonne gouvernance des entreprises et des administrations. À cet égard, ceux qui estiment pouvoir les relier à une néolibéralisation des politiques culturelles pêchent par excès de simplification. Ce n'est pas que la thèse soit totalement inexacte. Mais, aveuglée par la cohérence d'un projet politique, elle ne rend pas assez compte de l'incohérence même des rapports de pouvoir au sein des politiques culturelles. La différenciation de nature et d'intensité de contrôle que nous notons entre le secteur du patrimoine et celui qui touche à la démocratisation culturelle peut, jusqu'à un certain point, être interprétée en ce sens. Le patrimoine serait alors ce secteur (conservateur par essence) que les libéraux excluent volontiers du marché ; tandis que la démocratisation culturelle (plus progressiste) viserait des acteurs et des dispositifs plus facilement transférables au marché, quand on ne les écarte pas, tout simplement, d'un financement public soucieux de rigueur. Mais l'axe libéral/keynésien n'est pas le plus pertinent pour comprendre le sens de la recherche, par les instances centrales du ministère, de nouvelles marges de manœuvre à l'égard des échelons déconcentrés (les DRAC). Il n'est pas non plus le plus intéressant pour comprendre l'agencification des politiques culturelles régionales et leur investissement dans la concertation institutionnelle.

Des différences apparaissent d'abord entre ces processus. La suite normative (LOLF - RGPP) et le logiciel Chorus sont vus comme des menaces pour les DRAC dont la montée en puissance semble mise en cause. A l'inverse, les instruments que nous avons pointés dans l'examen des interventions régionales contribuent plutôt à en conforter leurs responsabilités en matière culturelle alors qu'elles peinent à s'imposer aux côtés de collectivités publiques plus fortement et financièrement impliquées.

11 Concertation nationale initiée dans le champ du spectacle vivant, entre 2008 et 2009, par le ministère de la Culture, avec les organisations professionnelles du secteur et des représentants des différents échelons de collectivités territoriales. 
Mais la dimension politique de ce tournant instrumental est ailleurs. Dans les deux cas, son analyse dévoile des enjeux de légitimation au sein de rapports de force internes et externes aux institutions. Les transformations qui touchent à l'État se justifient à la fois par la recherche de nouvelles capacités d'influence du ministère sur ses directions régionales, et la velléité, de la part du ministère des Finances, d'un contrôle resserré sur un domaine, la culture, considérée (péjorativement) comme un foyer de dépense. L'inflation de procédures de contrôle, et la tension qui existe entre elles, s'expliquent en interne et en externe par ces rapports de force. C'est en les prenant en compte que s'éclairent les incohérences entre objectifs initiaux et efficience des outils.

À l'échelle des régions, il s'agit de faire de la culture un domaine légitime des politiques régionales, ce qu'il est de façon très inégale selon les régions. Le rapport de force se situe dès lors entre les directions à la Culture (et leurs élus) et les autres segments de l'administration régionale et de son exécutif politique. La rationalisation qu'opère une agencification, et les ressources politiques que peut produire une concertation bien conduite, sont de nature à accréditer la culture comme secteur légitime ou politiquement rentable d'intervention publique. Mais ces changements se comprennent aussi dans le cadre multi-niveaux de l'action publique culturelle. L'entreprise de légitimation qu'ils représentent s'adressent aussi à l'État, aux métropoles et départements (échelon plus important que les régions en matière culturelle). Ces changements instrumentaux s'inscrivent dans une période d'intense réflexion autour des politiques culturelles du XXI ${ }^{\text {ème }}$ siècle à l'échelle internationale (Bonet et Négrier 2008 et 2011, Audet et Saint-Pierre 2010, Poirrier 2011). Dans une situation encore hybride où se mêlent l'ancien et le nouveau, innovations et continuités, la lecture instrumentale des politiques culturelles est peut-être la seule clé de lecture possible du changement, en l'absence de groupes sociaux suffisamment puissants pour traduire un tournant d'une autre ampleur, un nouveau référentiel de politiques publiques.

\section{Références citées :}

Audet, C. \& Saint-Pierre, D. (2011, dir.), Tendances et défis des politiques culturelles, Québec : Presses de l'Université Laval

Barrère, A. (2010), « Ce que fait l'évaluation aux établissements scolaires. Une année dans un collège d' " éducation prioritaire » », Ethnologie Française, vol. XL, n¹, p.141-149

Belorgey, N. (2010), L'hôpital sous pression. Enquête sur le nouveau management public, Paris : La Découverte

Bezes P. (2005), «Le renouveau du contrôle des bureaucraties » L'impact du New Public Management, Informations sociales, 6, $\mathrm{n}^{\circ} 126, \mathrm{p} .26-37$.

Bezes, P. (2009), Réinventer l'État. Les réformes de l'administration française (1962-2008), Paris, PUF, coll. Le lien social

Bodiguel, J.-L. (2000), "Naissance et affirmation des directions régionales des affaires culturelles », in. Ph. Poirrier, J.P. Rioux, (dir.), Affaires culturelles et territoires, Comité d'histoire du ministère de la culture, Paris : La Documentation Française, p.21-54

Bonet, L., Négrier, E. (2008, dir.), La fin des cultures nationales ?, Paris : La Découverte Bonet, L., Négrier, E., 2011, «The End(s) of National Cultures? Cultural Policy in the Face of Diversity », International Journal of Cultural Policy, Vol.17, $\mathrm{n}^{\circ} 1$, p.1-16

Bouët, J. (2010), 21 propositions pour relancer le partenariat entre l'État et les collectivités territoriales dans le domaine culturel, Paris : Ministère de la culture et de la communication 
Cardona, J., Lacroix, C. (2002), Chiffres clefs 2001, Paris, Ministère de la Culture, Paris : Documentation Française.

Dardy-Chrétien, M. (2007), Michel Guy, Secrétaire d'État à la culture, 1974-1976. Un innovateur méconnu, Comité d'histoire du ministère de la culture, La Documentation Française

DEPS (2011), (Département des Études, de la Prospective et des Statistiques), Culture \& Médias 2030, Questions de culture, Paris : Ministère de la Culture et de la Communication 2011.

Djakouane, A., Négrier, E. (2010), La Culture pour chacun en Languedoc-Roussillon, Rapport pour le ministère de la Culture, OPPIC, 56 p.

Élias, N., Stocson, J. C. (1997), Logiques de l'exclusion, Paris : Fayard

Epstein, R. (2005), « Gouverner à distance. Quand l'État se retire des territoires », Esprit, $\mathrm{n}^{\circ}$ 319, p. 96-111.

Jobert, B., Muller P. (1987), L'État en action, PUF, 1987.

Jobert B., «Représentations sociales, controverses et débats dans la conduite des politiques publiques », Revue Française de Science Politique, 42 (2), avril 1992, p. 219-234.

Lascoumes, P. (2007), «La Gouvernementalité : de la critique de l'État aux technologies du pouvoir », Le Portique [En ligne] , 13-14|2004, mis en ligne le 15 juin 2007, Consulté le 27 juin 2011. URL : http://leportique.revues.org/index625.html

Lascoumes P., Le Galès P., 2004a) «Instruments », in. L. Boussaguet, S. Jacquot, P. Ravinet, Dictionnaire des Politiques Publiques, Presses de Sciences Po, 2004, p. 267-270.

Lascoumes, P., Le Galès P. (2004b), «L'action publique saisie par les instruments », in Lascoumes P., Le Galès P., Gouverner par les instruments, Presses de Sciences Po, p. 11-44.

Lucas, J.M., (2012) Culture et Développement Durable, Paris : IRMA, coll. Revolutic

Menger, P.-M. (2005), Les intermittents du spectacle. Sociologie d'une exception, Paris : Éditions de l'EHESS

Moulinier P. (2002), Politique culturelle et décentralisation, Paris, L'Harmattan

Moulinier, P. (2010), «Le Patrimoine », dans Ph. Poirrier (dir.), Politiques et pratiques de la culture, Paris : Documentation française, p.79-89

Négrier, E., Teillet, P. (2011), La question régionale en culture, in S. Barone (dir.), Les politiques régionales en France, La Découverte - Pacte, p. 135-160.

Négrier, E., Teillet, P. (2014), «La réforme de l'État culturel local. Reconcentration instrumentale ou 'contrôle orienté' », Sciences de la Société, n90, p. 77-92.

Négrier, E., Valarié, P. (1997), "Patrimoines en ligne. La mémoire comme objet industriel" Sciences de la Société ${ }^{\circ} 40, \mathrm{p} .157-175$

Négrier, E. (2008), «Public Policies for the Performing Arts in France », in C. Smithuijsen (ed.) State on Stage, Amsterdam : BoekmanStudies, p.79-89

Négrier, E., dir. (2002), Patrimoine culturel et décentralisation, Paris : L'Harmattan, Logiques Politiques

Patriat, C. (2010), Le ministère de la Culture au fourneau des réformes, dans $\mathrm{Ph}$. Poirrier (dir.), Politiques et pratiques de la culture, Paris : Documentation française, p.23-34

Poirrier, P. (2011, dir.), Regards sur une histoire des politiques culturelles dans le monde.

Études de cas. 1945-2011, Paris, La Documentation française

Pôle Sud, (1999), Les politiques culturelles en Europe du Sud, $\mathrm{n}^{\circ} 10$

Pongy, M. (2004), «L'intervention des régions et des départements », in G. Saez (dir.), Institutions et vie culturelles, Paris : La Documentation Française, p. 50-54.

Pontier, J.-M. (2003), Rapport d'évaluation des protocoles de décentralisation culturelle, Ministère de la Culture et de la Communication.

Pontier, J.-M., Ricci J.-C. Bourdon J. (1996), Droit de la culture, Paris : Dalloz, $2^{\text {ème }}$ éd. 
Rauch, M.-A. (1998), Le bonheur d'entreprendre. Les administrateurs de la France d'outremer et la création du ministère des affaires culturelles, Paris, La Documentation Française, Comité d'Histoire du ministère de la Culture.

Ritaine E. (1983) Les stratèges de la culture, Paris : Presses de la Fondation Nationale des Sciences Politiques

Rouban, L. (2008), «La réforme de l'État et son contexte - Réformer l'État pourquoi et pour quoi ? », in Cahiers Français, n 346 , septembre-octobre, p. 3-7.

Rozier, S. (2010), «La culture à l'encan», dans L. Bonelli et W. Pelletier (dir.), L'État démantelé. Enquête sur une révolution silencieuse, Paris: La Découverte-Le Monde Diplomatique, p.274-283

Teillet, P. (2004), “La politique des politiques culturelles", L'Observatoire, $\mathrm{n}^{\circ} 25$, p. 4-10.

Urfalino, P. (1996), L'invention de la politique culturelle, Paris : la Documentation Française 